\title{
Use of Waist Circumference and Ultrasonographic Assessment of Abdominal Fat Distribution in Predicting Metabolic Risk Factors in Healthy Japanese Adults
}

\author{
Takaaki Kondo $^{1)}$, Maimi Abe ${ }^{2)}$, Jun Ueyama ${ }^{1)}$, Akiko Kimata ${ }^{1)}$, Kanami Yamamoto ${ }^{1)}$ and Yoko Hori ${ }^{3)}$ \\ 1) Program in Radiological and Medical Laboratory Sciences, Nagoya University Graduate School of Medicine \\ 2) Nagoya Memorial Hospital \\ 3) Program in Nursing, Nagoya University Graduate School of Medicine
}

\begin{abstract}
The purpose of this study is to evaluate the association of abdominal fat thickness with obesity-associated metabolic risk factors. A total of 186 men and 350 women aged 30-78, who were voluntary participants in a communitybased health screening program, were examined for body mass index (BMI), waist circumference (WC), and WC-stature ratio (WCSR). Using the ultrasonographic method, the maximum thickness of the preperitoneal fat layer (Pmax) and the minimum and maximum thicknesses of the subcutaneous fat layer (Smin and Smax) in the abdominal region were measured and the abdominal wall fat index $(\mathrm{AFI}=\mathrm{Pmax} / \mathrm{Smin})$ was calculated. Each measurement was used as a main predictor in the logistic model where the presence of a metabolic risk factor (high blood pressure, high triglyceride, low high-density lipoprotein cholesterol, high glucose, and high uric acid) was predicted. Common covariates in the models were age, smoking status, drinking habit, and overall physical activity. Both WC and WCSR, which behaved in the same manner as BMI, showed a significant association with the majority of metabolic risk factors, but these anthropometric indices were not independent of the confounding effect of BMI. In relationship with atherogenic dyslipidemia, Smax in women and Pmax in both men and women were significant predictors independent of BMI. AFI was also found to be a BMIindependent predictor of atherogenic dyslipidemia in both genders. In men, AFI and high glucose were not significantly associated; in women, they were inversely associated. No measurement showed a significant association with high uric acid. Results suggest that ultrasonographic evaluation of abdominal fat is useful for identifying those at atherogenic risk. A larger sample of subjects in terms of body composition may be required to confirm the usefulness of ultrasonographic evaluation of high glucose. J Physiol Anthropol 28(1): 7-14, 2009 http://www.jstage.jst.go.jp/browse/jpa2
\end{abstract}

[DOI: 10.2114/jpa2.28.7]

Keywords: body mass index, visceral fat, preperitoneal fat, abdominal wall fat index, health screening, dyslipidemia, logistic model, ROC curve

\section{Introduction}

For over 50 years, a close association of centralized obesity with diabetes, atherosclerosis, gout, and uric calculous disease has been repeatedly underscored (Vague, 1956). Since then, numerous studies have confirmed the increased risk for cardiovascular disease, hypertension, metabolic complications, and mortality due to centrally located body fat (Blair et al., 1984; Folsom et al., 1993; Hartz et al., 1984; Katzel 1993; Kissenbah et al., 1982). While the pattern of fat distribution has traditionally been determined by measurement of triceps and subscapular skinfold thicknesses, waist circumference (WC), which has been widely used to measure the degree of central fat distribution (Schwingel, 2007a), is known to be more closely linked to cardiovascular disease (CVD) risk factors than is body mass index (BMI) (Zhu et al., 2002). Although WC is likely correlated with abdominal fat volume (Kvist et al., 1988), subcutaneous fat accumulation in the abdominal region cannot be distinguished from intraabdominal visceral fat accumulation, which plays a major role in the development of metabolic syndrome or CVD (Fujioka et al., 1987; Kissenbah, 1996).

To overcome this methodological limitation of the anthropometric measurement, the use of ultrasonography to evaluate abdominal fat has been suggested as an alternative technique. The ultrasonographic method is highly useful because of the low cost, absence of x-ray exposure, and high correlation with radiographic computed tomography (CT) (Armellini et al., 1990; Leite et al., 2002). Recently, Suzuki et al. (1993) demonstrated that intra-abdominal fat thickness determined by ultrasonography evidenced a significant correlation with the presence of CVD risk factors, including high blood pressure, dyslipidemia, hyperglycemia, and 
hyperinsulinemia. Although their report emphasized the value of ultrasonographic measurement of subcutaneous and intraabdominal fat in evaluating metabolic risk, the selected study population included relatively overweight subjects (mean, 28.0 $\mathrm{kg} / \mathrm{m}^{2}$; range, 20.3-42.9), the majority ( $82 \%$ ) of whom were diagnosed as hyperlipidemic. Hence, the usefulness of ultrasonographic measurement of abdominal fat has been little documented in identifying those who are susceptible to metabolic complications in general populations. For this purpose, we conducted a cross-sectional study to collect data on the anthropometric and ultrasonographic measurements of abdominal fat accumulation, each of which was evaluated for its association with metabolic risk factors.

\section{Methods}

The present study was conducted in 1999 with the cooperation of Nishibiwajima Town, a suburban municipality with a population of 16,000 located in central Japan. A total of 247 men and 428 women participated in an annual health screening program for residents of the town at two community centers. The participants underwent a physical examination including height and weight for calculation of BMI $\left(\mathrm{kg} / \mathrm{m}^{2}\right)$, blood pressure measurements, and a fasting blood sample collection for determining serum lipid and plasma glucose concentrations. The participants were asked to respond to a self-reported questionnaire covering medical history, smoking and drinking status, and other lifestyle characteristics. In addition to the ordinary anthropometric measurements, we examined the distribution pattern of abdominal fat by measuring waist circumference (WC) and the thicknesses of abdominal fat layers. WC, defined as the circumference in centimeters at the umbilical level, was obtained using a flexible, plastic tape measure with the subject breathing normally in a standing position. The accuracy of the WC measurement was set to $0.5 \mathrm{~cm}$. Because $\mathrm{WC}$ was considered to vary with body height, we adjusted for the effect of height by calculating the WC-stature ratio (WCSR).

Abdominal fat pad thickness was assessed by an ultrasonographic measurement using SonoAce 600 (Medison Japan) according to the method of Suzuki et al. (1993). Using the linear-array probe $(7.5 \mathrm{MHz} / 40 \mathrm{~mm})$ kept perpendicular to the skin on the upper median abdomen, longitudinal scanning was done from the xiphoid process to the umbilicus along the linea alba of the subject in a supine position. The probe was maintained to make contact as lightly as possible so as not to compress the fat layer. The maximum thickness of preperitoneal fat (Pmax), and the minimum and maximum thicknesses of subcutaneous fat (Smin and Smax, respectively) were measured in millimeters directly from the frozen images on the screen with electronic calipers (Fig. 1). WC and ultrasonographic measurements were made by a trained nurse and physician, respectively, throughout the study. Moreover, the abdominal wall fat index (AFI) was calculated as the Pmax/Smin ratio, resulting in acquisition of seven fatness-

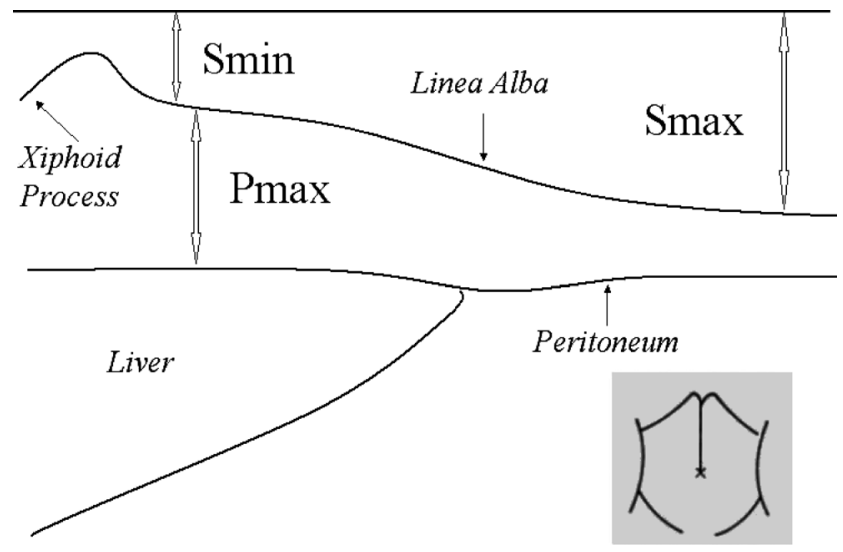

Fig. 1 Vertical view of abdominal region along the midline. Smin, minimum thickness of subcutaneous fat pad. Pmax, maximum thickness of preperitoneal fat pad. Smax, maximum thickness of subcutaneous fat pad.

related indices in total (i.e., BMI, WC, WCSR, Smin, Pmax, Smax and AFI), 6 of which were later used as predictor variables in the logistic regression model.

In accord with the ATP III report (Expert Panel on Detection Evaluation and Treatment of High Blood Cholesterol in Adults, 2001; Park, 2004), we defined obesity-associated metabolic risk factors as follows: 1) high blood pressure (BP) as a systolic blood pressure (SBP) $\geq 140 \mathrm{mmHg}$ or a diastolic blood pressure (DBP) $\geq 90 \mathrm{mmHg} ; 2$ ) high triglyceride (TG) as a concentration $\geq 1.69 \mathrm{mmol} / \mathrm{L}(150 \mathrm{mg} / \mathrm{dL})$; 3) low highdensity lipoprotein cholesterol (HDL-C) as a concentration $<1.03 \mathrm{mmol} / \mathrm{L}(40 \mathrm{mg} / \mathrm{dL})$ for men and $<1.29 \mathrm{mmol} / \mathrm{L}(50$ $\mathrm{mg} / \mathrm{dL}$ ) for women; and 4) high glucose as a fasting plasma glucose concentration $\geq 6.10 \mathrm{mmol} / \mathrm{L}(110 \mathrm{mg} / \mathrm{dL})$. Because hyperuricemia, though not required for the definition of metabolic syndrome, is thought to be part of metabolic syndrome (Zimmet et al., 2001), we added the following components: 5) high uric acid (UA) as a concentration $\geq 0.417$ $\mathrm{mmol} / \mathrm{L}(7.0 \mathrm{mg} / \mathrm{dL})$ for men and $\geq 0.327 \mathrm{mmol} / \mathrm{L}(5.5 \mathrm{mg} / \mathrm{dL})$ for women. Each of these metabolic risk factors was treated as a binary health outcome in the statistical model.

For definition of covariates, smoking status was categorized as current, past, and never, and drinking as current and other; overall physical activity was categorized into three levels as active, moderately active, and inactive. Age in years was also used as a covariate. Gender-specific covariate-adjusted odds ratios (ORs) and 95\% confidence intervals (CIs) were calculated for association with BMI as a main predictor variable. BMI was then replaced alternately with either $\mathrm{WC}$, WCSR, Smax, Pmax, or AFI (Model-1). Furthermore, BMI was included as an additional covariate in the logistic model where WC, WCSR, Smax, Pmax, or AFI were treated as main predictors (Model-2). Estimated ORs were customized in all models to correspond to an increment of $1 \mathrm{SD}$ which was calculated using the gender-specific whole population data. This customization was performed to promote comparability of ORs between different logistic models. In order to evaluate 
the diagnostic validity of those fatness-related indices in the logistic regression models, we calculated the areas under the receiver operating characteristic (ROC) curve (AUCs) after adjustment for the effects of covariates. We calculated the $95 \%$ CIs of the AUC based on the nonparametric percentile method, using a bootstrap approach with 1,000 bootstrap samples and without bias corrections (Davison et al., 2003).

Of the 675 participants who consented to the use of their personal information for data analysis, 139 were not included in the statistical analyses because of missing answers to lifestyle questions, medical history of hypertension, diabetes, dyslipidemia, or malignancy, or insufficient blood sample volume for biochemical assay. Consequently, data from 186 men and 350 women were analyzed separately. We conducted all data storage and analyses using the Statistical Analysis System (SAS) release 9.1 licensed to the Nagoya University Information Technology Center.

The current study protocol was approved by the Ethical Board of the Nagoya University Graduate School of Medicine.

\section{Results}

The basic characteristics of the subjects and the prevalence of obesity-associated metabolic risk factors are shown in Table

Table 1 Basic characteristics of the study population and prevalence of abnormal metabolic profiles

\begin{tabular}{|c|c|c|}
\hline & Men $(n=186)$ & Women $(n=350)$ \\
\hline & Mean (Range) & Mean (Range) \\
\hline Age (year) & $54.5(30-77)$ & $53.6(30-78)$ \\
\hline \multicolumn{3}{|l|}{ Blood Pressure (mmHg) } \\
\hline Systolic & $122.1(88-206)$ & $117.1(78-185)$ \\
\hline Diastolic & $78.6(50-123)$ & $74.1(40-110)$ \\
\hline Triglyceride (mmol/L) & $1.4(0.32-5.9)$ & $1.1(0.28-5.2)$ \\
\hline HDL cholesterol (mmol/L) & $1.4(0.72-2.6)$ & $1.6(0.72-2.7)$ \\
\hline Glucose $(\mathrm{mmol} / \mathrm{L})$ & $5.4(3.8-15.5)$ & $5.2(4.2-8.8)$ \\
\hline BMI $\left(\mathrm{kg} / \mathrm{m}^{2}\right)$ & $23.1(16.6-34.1)$ & $22.2(16.4-34.4)$ \\
\hline $\mathrm{WC}(\mathrm{cm})$ & $81.1(59.0-118.0)$ & $70.7(56.0-114.0)$ \\
\hline WCSR & $0.49(0.36-0.66)$ & $0.46(0.36-0.77)$ \\
\hline $\operatorname{Smax}(\mathrm{mm})$ & $20.2(4-52)$ & $25.4(6-52)$ \\
\hline $\operatorname{Pmax}(\mathrm{mm})$ & $10.0(3-25)$ & $10.3(3-23)$ \\
\hline \multirow[t]{2}{*}{ AFI } & $0.79(0.30-1.9)$ & $0.58(0.21-1.2)$ \\
\hline & Frequency $(\%)$ & Frequency $(\%)$ \\
\hline \multicolumn{3}{|l|}{ Age } \\
\hline$\geq 65(\mathrm{y} / \mathrm{o})$ & $50(26.9)$ & $76(21.7)$ \\
\hline \multicolumn{3}{|l|}{ Body mass index } \\
\hline$\geq 25 \mathrm{~kg} / \mathrm{m}^{2}$ & $44(23.7)$ & $58(16.6)$ \\
\hline \multicolumn{3}{|l|}{ Smoking status } \\
\hline Never & $62(33.3)$ & $320(91.4)$ \\
\hline Past & $44(23.7)$ & $10(2.9)$ \\
\hline Current & $80(43.0)$ & $20(5.7)$ \\
\hline \multicolumn{3}{|l|}{ Drinking habit } \\
\hline Yes & $131(70.4)$ & $129(36.9)$ \\
\hline Abnormal metabolic profiles & Frequency $(\%)$ & Frequency $(\%)$ \\
\hline \multicolumn{3}{|l|}{ High blood pressure } \\
\hline Systolic blood pressure $\geq 130 \mathrm{mmHg}$ or diastolic blood pressure $\geq 85 \mathrm{mmHg}$ & $71(38.2)$ & $94(26.9)$ \\
\hline \multicolumn{3}{|l|}{ High triglyceride } \\
\hline Triglyceride $\geq 1.69 \mathrm{mmol} / \mathrm{L}$ & $47(25.3)$ & $41(11.7)$ \\
\hline \multicolumn{3}{|l|}{ Low HDL-cholesterol } \\
\hline \multicolumn{3}{|l|}{ HDL-cholesterol $<1.03 \mathrm{mmol} / \mathrm{L}$ for men or } \\
\hline HDL-cholesterol $<1.29 \mathrm{mmol} / \mathrm{L}$ for women & $28(15.1)$ & $56(16.0)$ \\
\hline \multicolumn{3}{|l|}{ High glucose } \\
\hline Glucose $\geq 6.10 \mathrm{mmo} / \mathrm{L}$ & $71(38.2)$ & $24(6.9)$ \\
\hline \multicolumn{3}{|l|}{ High uric acid } \\
\hline $\begin{array}{l}\text { Uric acid } \geq 0.417 \mathrm{mmol} / \mathrm{L} \text { for men or } \\
\text { uric acid } \geq 0.327 \mathrm{mmol} / \mathrm{L} \text { for women }\end{array}$ & $56(30.1)$ & $58(16.6)$ \\
\hline
\end{tabular}

BMI, body mass index; WC, waist circumference; WCSR, WC-stature ratio; Smin, minimum thickness of subcutaneous fat pad; Pmax, maximum thickness of preperitoneal fat pad; Smax, maximum thickness of subcutaneous fat pad; AFI, abdominal wall fat index. 
Table 2 OR $(95 \% \mathrm{CI})$ and AUC $(95 \% \mathrm{CI})^{\S}$ associated with every SD increment of each anthropometic measurement in the logistic regression model predicting presence of metabolic risk factors

\begin{tabular}{|c|c|c|c|c|c|c|}
\hline \multirow{2}{*}{ Metabolic risk facto } & & \multirow{2}{*}{ BMI } & \multicolumn{2}{|c|}{ WC } & \multicolumn{2}{|c|}{ WCSR } \\
\hline & & & Model-1 & Model-2 & Model-1 & Model-2 \\
\hline \multirow[t]{2}{*}{ High blood pressure } & OR & $1.9(1.3-2.7)$ & $1.7(1.2-2.4)$ & $0.99(0.54-1.8)$ & $1.5(1.1-2.2)$ & $0.63(0.28-1.3)$ \\
\hline & AUC & $0.73(0.67-0.81)$ & $0.70(0.65-0.79)$ & $0.73(0.67-0.82)$ & $0.70(0.64-0.79)$ & $0.73(0.68-0.82)$ \\
\hline High triglyceride & OR & $1.8(1.3-2.7)$ & $1.9(1.3-2.8)$ & $1.4(0.75-2.7)$ & $2.0(1.3-3.0)$ & $1.6(0.77-3.4)$ \\
\hline Low HDL cholesterol & AUC & $0.70(0.65-0.82)$ & $0.69(0.62-0.81)$ & $0.70(0.65-0.83)$ & $0.69(0.63-0.81)$ & $0.71(0.65-0.82)$ \\
\hline \multirow[t]{2}{*}{ High glucose } & OR & $1.4(0.78-2.4)$ & $1.2(0.72-2.1)$ & $0.91(0.35-2.1)$ & $1.2(0.68-2.0)$ & $0.67(0.19-1.9)$ \\
\hline & AUC & $0.77(0.71-0.89)$ & $0.77(0.71-0.89)$ & $0.77(0.71-0.89)$ & $0.77(0.71-0.88)$ & $0.77(0.71-0.89)$ \\
\hline \multirow[t]{2}{*}{ High uric acid } & OR & $1.5(1.1-2.1)$ & $1.6(1.1-2.3)$ & $1.4(0.74-2.5)$ & $1.6(1.1-2.3)$ & $1.5(0.73-3.1)$ \\
\hline & AUC & $0.74(0.68-0.83)$ & $0.74(0.69-0.83)$ & $0.74(0.69-0.84)$ & $0.74(0.69-0.83)$ & $0.74(0.69-0.84)$ \\
\hline \multicolumn{7}{|l|}{ Women $(n=350)$} \\
\hline \multirow[t]{2}{*}{ Low HDL cholesterol } & OR & $1.7(1.3-2.3)$ & $1.7(1.3-2.3)$ & $1.3(0.76-2.3)$ & $1.6(1.2-2.2)$ & $1.0(0.56-1.8)$ \\
\hline & AUC & $0.71(0.67-0.79)$ & $0.73(0.67-0.80)$ & $0.73(0.68-0.80)$ & $0.72(0.66-0.79)$ & $0.72(0.67-0.80)$ \\
\hline \multirow[t]{2}{*}{ High glucose } & OR & $1.8(1.2-2.6)$ & $1.8(1.2-2.7)$ & $1.6(0.81-3.2)$ & $1.7(1.1-2.5)$ & $1.2(0.48-2.3)$ \\
\hline & AUC & $0.76(0.69-0.86)$ & $0.80(0.73-0.87)$ & $0.79(0.73-0.88)$ & $0.78(0.71-0.86)$ & $0.77(0.71-0.86)$ \\
\hline \multirow[t]{2}{*}{ High uric acid } & OR & $1.6(1.2-2.1)$ & $1.6(1.2-2.1)$ & $1.3(0.79-2.3)$ & $1.6(1.2-2.2)$ & $1.2(0.67-2.2)$ \\
\hline & AUC & $0.70(0.65-0.78)$ & $0.71(0.65-0.79)$ & $0.71(0.66-0.79)$ & $0.71(0.65-0.79)$ & $0.71(0.66-0.79)$ \\
\hline
\end{tabular}

OR, odds ratio; AUC, area under the curve; CI, confidence interval; SD, standard deviation; BMI, body mass index; WC, waist circumference; WCSR WCstature ratio; HDL, high-density lipoprotein

${ }^{\$}$ Estimated using a nonparametric bootstrap method

1. Both men and women distributed around the nearly ideal mean BMI of 23.1 and $22.2 \mathrm{~kg} / \mathrm{m}^{2}$, with those with BMI $\geq 25$ $\mathrm{kg} / \mathrm{m}^{2}$ accounting for $23.7 \%$ and $16.6 \%$, respectively. Ages ranged widely for both men and women and did not differ significantly on average between men and women. Mean BP, both diastolic and systolic, was significantly higher for men than for women. TG and glucose were significantly higher for men than for women, while HDL-C was significantly lower for women than for men. Men had higher abdominal adiposity than women, as indicated by significantly higher WC and WCSR, whereas the ultrasonographic measurements showed significantly thicker abdominal fat layers for women than for men in Smax and Pmax. AFI was significantly higher in men than in women. The most prevalent of all the obesityassociated risk factors was high BP for both men and women and high glucose for men, followed by high UA. Only $\approx 7 \%$ of women carried high glucose.

In the logistic model predicting the presence of high BP (Table 2), every SD increment of BMI was significantly associated with the estimated ORs of 1.9 (95\% CI: 1.3-2.7) and 2.0 (95\% CI: 1.5-2.7) for men and women, respectively. Likewise, a significant regression to the presence of risk factors was observed for the increase of WC and WCSR, with the respective ORs of 1.7 (95\% CI: 1.2-2.4) and 1.5 (95\% CI: 1.1-2.2) in men, and 1.7 (95\% CI: 1.3-2.3) and 1.8 (95\% CI: 1.3-2.4) in women (Model-1). Similar significant relationships with anthropometric measurements were observed for high TG, low HDL-C, and high UA in men, and low HDL-C, high glucose, and high UA in women. However, BMI adjustment resulted in the disappearance of a significant relationship of all metabolic risk factors with either WC or WCSR (Model-2).

With respect to ultrasonographic measurements (Table 3), Smax was significantly associated with high BP after BMI adjustment (Model-2) (OR: 1.6, 95\% CI: 1.0-2.7) in men and with high TG (OR: 1.8, 95\% CI: 1.1-2.9) and low HDL-C (OR: 2.0, 95\% CI: 1.3-3.2) in women. As for the prediction of metabolic risk factors with Pmax in Model-2, significant relationships were observed for high $\mathrm{BP}$ (OR: 1.9, 95\% CI: 1.2-3.1), high TG (OR: 1.6, 95\% CI: 1.0-2.6), and low HDLC (OR: 1.9, 95\% CI: 1.1-3.4) in men, and low HDL-C (OR: 1.9, 95\% CI: 1.3-2.6) in women. Metabolic risk factors indicating a significant association with AFI in Model-2 was high TG (OR: 1.9, 95\% CI: 1.1-3.4) and low HDL-C (OR: 1.9, 95\% CI: 1.1-3.4) in men. In women, low LDL-C was also predicted by AFI with a significant OR of 1.4 (95\% CI: 1.0-1.8), but high glucose was inversely predicted with a significant OR of 0.6 (95\% CI: $0.33-0.99)$. In both men and women, none of the anthropometric and ultrasonographic measurements was associated with high uric acid in Model-2.

In the logistic model using BMI to predict high blood pressure, the AUCs were estimated at 0.73 (95\% CI: $0.57-0.81)$ and 0.79 (95\% CI: $0.75-0.84)$ for men and women, 
Table 3 OR $(95 \% \mathrm{CI})$ and AUC $(95 \% \mathrm{CI})^{\S}$ associated with every SD increment of each ultrasonic measurement in the logistic regression model predicting presence of metabolic risk factors

\begin{tabular}{|c|c|c|c|c|c|c|c|}
\hline \multirow{2}{*}{\multicolumn{2}{|c|}{ Metabolic risk factor }} & \multicolumn{2}{|c|}{ Smax } & \multicolumn{2}{|c|}{ Pmax } & \multicolumn{2}{|c|}{ AFI } \\
\hline & & Model-1 & Model-2 & Model-1 & Model-2 & Model-1 & Model-2 \\
\hline \multicolumn{8}{|l|}{$\operatorname{Men}(n=186)$} \\
\hline \multirow{2}{*}{ High blood pressure } & OR & $2.1(1.4-3.1)$ & $1.6(1.0-2.7)$ & $2.3(1.6-3.5)$ & $1.9(1.2-3.1)$ & $1.2(0.87-1.7)$ & $1.2(0.83-1.6)$ \\
\hline & AUC & $0.70(0.65-0.79)$ & $0.72(0.67-0.82)$ & $0.71(0.66-0.80)$ & $0.73(0.68-0.82)$ & $0.66(0.61-0.77)$ & $0.73(0.68-0.82)$ \\
\hline \multirow[t]{2}{*}{ High triglyceride } & OR & $1.3(0.93-1.9)$ & $0.80(0.49-1.3)$ & $2.0(1.4-3.0)$ & $1.6(1.0-2.6)$ & $1.6(1.2-2.3)$ & $1.6(1.1-2.3)$ \\
\hline & AUC & $0.64(0.60-0.76)$ & $0.70(0.66-0.81)$ & $0.71(0.64-0.81)$ & $0.72(0.67-0.83)$ & $0.68(0.62-0.79)$ & $0.73(0.67-0.83)$ \\
\hline \multirow[t]{2}{*}{ Low HDL cholesterol } & OR & $1.6(1.1-2.5)$ & $1.1(0.63-1.9)$ & $2.3(1.5-3.8)$ & $1.9(1.1-3.4)$ & $1.9(1.3-3.0)$ & $2.0(1.3-3.1)$ \\
\hline & AUC & $0.65(0.60-0.79)$ & $0.70(0.65-0.83)$ & $0.70(0.64-0.83)$ & $0.72(0.67-0.85)$ & $0.66(0.61-0.81)$ & $0.74(0.68-0.86)$ \\
\hline \multirow[t]{2}{*}{ High glucose } & OR & $1.2(0.61-2.2)$ & $0.95(0.43-2.0)$ & $1.2(0.64-2.1)$ & $0.97(0.48-1.9)$ & $1.2(0.73-2.0)$ & $1.2(0.71-2.0)$ \\
\hline & AUC & $0.77(0.70-0.89)$ & $0.78(0.71-0.90)$ & $0.78(0.70-0.89)$ & $0.78(0.72-0.90)$ & $0.77(0.71-0.89)$ & $0.78(0.72-0.89)$ \\
\hline \multirow[t]{2}{*}{ High uric acid } & OR & $1.4(0.99-2.1)$ & $1.1(0.71-1.9)$ & $1.6(1.1-2.4)$ & $1.4(0.88-2.2)$ & $1.1(0.77-1.5)$ & $1.1(0.74-1.5)$ \\
\hline & AUC & $0.74(0.67-0.83)$ & $0.74(0.69-0.84)$ & $0.74(0.67-0.83)$ & $0.74(0.69-0.83)$ & $0.72(0.66-0.82)$ & $0.78(0.72-0.89)$ \\
\hline \multicolumn{8}{|l|}{ Women $(\mathrm{n}=350)$} \\
\hline \multirow[t]{2}{*}{ High blood pressure } & OR & $1.7(1.3-2.3)$ & $1.1(0.78-1.7)$ & $1.4(1.1-1.8)$ & $1.0(0.74-1.4)$ & $0.87(0.66-1.1)$ & $0.88(0.66-1.2)$ \\
\hline & AUC & $0.77(0.73-0.83)$ & $0.79(0.75-0.84)$ & $0.75(0.71-0.81)$ & $0.79(0.75-0.84)$ & $0.74(0.70-0.80)$ & $0.73(0.68-0.82)$ \\
\hline \multirow[t]{2}{*}{ High triglyceride } & OR & $1.6(1.1-2.3)$ & $1.8(1.1-2.9)$ & $1.4(0.99-1.9)$ & $1.4(0.92-2.0)$ & $1.3(0.91-1.8)$ & $1.3(0.91-1.8)$ \\
\hline & AUC & $0.73(0.68-0.81)$ & $0.73(0.69-0.82)$ & $0.72(0.67-0.80)$ & $0.72(0.68-0.80)$ & $0.71(0.67-0.81)$ & $0.73(0.67-0.83)$ \\
\hline \multirow[t]{2}{*}{ Low HDL cholesterol } & OR & $2.2(1.6-3.1)$ & $2.0(1.3-3.2)$ & $2.1(1.5-2.9)$ & $1.9(1.3-2.6)$ & $1.3(1.0-1.8)$ & $1.4(1.0-1.8)$ \\
\hline & AUC & $0.74(0.70-0.82)$ & $0.74(0.70-0.82)$ & $0.76(0.70-0.82)$ & $0.76(0.72-0.83)$ & $0.67(0.61-0.76)$ & $0.74(0.68-0.86)$ \\
\hline \multirow[t]{2}{*}{ High glucose } & OR & $1.7(1.1-2.7)$ & $1.3(0.71-2.3)$ & $1.2(0.78-1.8)$ & $0.81(0.47-1.4)$ & $0.59(0.33-0.97)$ & $0.60(0.33-0.99)$ \\
\hline & AUC & $0.77(0.72-0.86)$ & $0.77(0.72-0.87)$ & $0.73(0.65-0.83)$ & $0.76(0.69-0.86)$ & $0.75(0.70-0.86)$ & $0.78(0.72-0.89)$ \\
\hline \multirow[t]{2}{*}{ High uric acid } & OR & $1.5(1.1-2.0)$ & $1.2(0.77-1.7)$ & $1.3(0.98-1.7)$ & $1.0(0.74-1.5)$ & $0.93(0.68-1.2)$ & $0.94(0.64-1.3)$ \\
\hline & AUC & $0.69(0.64-0.78)$ & $0.71(0.66-0.79)$ & $0.67(0.62-0.76)$ & $0.71(0.65-0.79)$ & $0.65(0.60-0.75)$ & $0.74(0.68-0.83)$ \\
\hline
\end{tabular}

OR, odds ratio; AUC, area under the curve; CI, confidence interval; Smax maximum thickness of subcutaneous fat pad; Pmax maximum thickness of preperitoneal fat pad; AFI, abdominal wall fat index; HDL, high-density lipoprotein

${ }^{\$}$ Estimated using a nonparametric bootstrap method

respectively; almost equivalent AUCs were estimated when other anthropometric or ultrasonographic measurements were used in Model-2, except for AFI in women $(0.73,95 \% \mathrm{CI}$ : 0.68-0.82). In Model-2 predicting high TG, low HDL-C, high glucose, or high uric acid, AUCs estimated using WC, WSR, or ultrasonographic measurements as the main predictor were found to be equal to or greater than the AUC estimated using BMI.

\section{Discussion}

Visceral fat accumulation in the abdominal region has been considered an important marker for assessing metabolic risks. Development of an inexpensive, convenient means of evaluating the abdominal fat volume has great practical implications for identifying those at high risk of metabolic syndrome in public health settings. Though CT scanning is the standard for determining visceral fat volume in the abdominal region (Kvist et al., 1988), it is not routinely used. WC or waist-to-hip ratio is often used to evaluate the pattern of body fat distribution (Shimokata et al., 1989a; Shimokata et al., 1989b; Shimokata et al., 1989c). Though WC has been known to serve as a useful marker of intra-abdominal fat and a strong correlate of visceral adipose tissue, there is a report questioning its accuracy (Ross et al., 1996). To compensate for this methodological limitation, we employed ultrasonographic measurement of abdominal fat thickness. Whereas there are some variations in determining the anatomical sites to quantify the fat size, we conformed to the procedure proposed by Suzuki et al. (1993).

The statistical method employed in this study was logistic regression analysis. Another possible approach is the use of an analysis-of-covariance (ANCOVA) model, in which dependent variables are treated as continuous. We found that the ANCOVA was more sensitive than the logistic regression analysis in terms of statistical power in detecting significant relationships (data not shown). However, we did not employ the ANCOVA to model association, since our primary purpose was not to examine linear relationships but to evaluate the effect of fatness on the presence of the metabolic risks and related diagnostic validity.

In a previous study, it was pointed out that use of BMI alone does not account for the wide variation in body fat at any level of body size (Michels et al., 1998), calling into question the usefulness of BMI as a predictor for identifying those having risk factors. However, our study showed a strong link between BMI and the majority of metabolic risk factors in both men and women, indicating the potential usefulness of BMI to distinguish individuals at risk for metabolic syndrome. We also found a significant relationship between other anthropometric determinations (WC and WCSR) and metabolic risk factors, consistent with previous reports (Han et al., 1995; Hartz et al., 
1984; Katzel et al., 1993). The relatively similar magnitude of ORs associated with every SD increment of these anthropometric measurements means that WC or WCSR is as useful as BMI in predicting the presence of metabolic risk factors. However, these measurements did not seem to be independent of the effect of BMI because the significant relationship disappeared in Model-2, suggesting that BMI is a strong confounder.

Our results showed that ultrasonographic measurements behave in a manner somewhat different from anthropometric ones. There are three major zones of adipose tissue, i.e., superficial subcutaneous adipose tissue (SSAT), deep subcutaneous adipose tissue (DSAT) which is delineated by fascial plane, and visceral or intra-abdominal adipose tissue (VAT) (Sniderman et al., 2007). In this study, we considered that Smax represents the SSAT thickness in the abdominal region, while Pmax stands for the thickness of DSAT which shares common morphologic and functional properties with VAT. Of these three adipose tissue components, SSAT has been known to be least associated with atherogenic dyslipidemia or dysglycemia to date. Our results revealed a significant relationship of Smax with both high TG and low HDL-C in Model-1 and Model-2 for women, whereas there was no such significant relationship in men, suggesting subcutaneous fat accumulation is an independent predictor of atherogenic dyslipidemia in women. Regarding the relationship Pmax with atherogenic risk factors, high $\mathrm{BP}$, high TG, and low HDL-C were well predicted in men, and low HDL-C in women, consistent with numerous lines of evidence that DSAT has as strong an association with atherogenic dyslipidemia as VAT (Sniderman et al., 2007). Limited evidence has suggested that greater transmembrane fluxes of fatty acids are associated with dyslipidemia (Marin et al., 1992); greater efflux of fatty acids in the upper body to the liver results in greater hepatic TG and cholesterol synthesis (Sniderman et al., 1998). Besides, smaller TG-rich HDL particles produced as a result of increased secretion of very-low-density lipoprotein by the liver tend to be cleared more rapidly than the larger ones (Sniderman et al., 2002).

Taken together, our results support our assumption that the ultrasonographic measurement of preperitoneal fat accumulation is useful in identifying those who are at risk for atherogenic complications as well in a general healthy population as among patients with clinical dyslipidemia. In addition, this assumption was also corroborated by the results of diagnostic validity examination (Fletcher et al., 1999), which demonstrated that the ultrasonographic measurements distinguished as well as or better than BMI between those at high metabolic risk and the others, thus indicating their satisfactory sensitivity and specificity. A new possibility must also be borne in mind that SSAT in the abdominal region is also an independent marker identifying women at risk for atherogenic disease. Although the reason for the disparity between our finding and the previous studies reporting that SSAT is only moderately associated with atherogenic dyslipidemia is not clear, we presume that trunk SSAT contributes to some extent to the development of atherosclerosis in women. This speculation is supported in part by the previous literature reporting that not only visceral but also truncal adiposity seems to be related to increased arterial stiffness, and that accumulation of adipose tissue preferentially in the trunk has more adverse effects on cardiovascular risk than does fat stored in peripheral depots (Ferreira et al., 2004).

A significant correlation of AFI with the ratio of the visceral fat area to the subcutaneous fat area determined by radiographic CT among patients with hyperlipidemia or glucose intolerance was already confirmed (Suzuki et al., 1993). However, the usefulness of AFI seems to depend on the situation in which it is applied. A previous paper demonstrated no significant correlation of AFI with risk factors for atherosclerosis in nonobese men (Yamamoto et al., 1997), while another report indicated the usefulness of AFI in evaluating disorders of metabolism among admitted patients with BMI $\geq 22 \mathrm{~kg} / \mathrm{m}^{2}$ (Kawamoto et al., 2002). We believe that this disagreement is due largely to differences in population characteristics between studies. Our results suggest that AFI predicts those at high risk of atherogenic dyslipidemia in the general population. Furthermore, relationships between AFI and metabolic risk factors were not affected by the confounding effects of BMI. The estimated ORs and 95\% CI before and after adjustment for BMI closely overlapped. This supports the usefulness of AFI for evaluating atherogenic risk in the general healthy population.

Expansion of DSAT and VAT has been repeatedly linked to an increased risk of dysglycemia (Sniderman et al., 2007). However, our results failed to show significant associations between high glucose and fatness; a lack of such significant associations was also confirmed in the ANCOVA model (data not shown). To our knowledge, the concentration of fasting plasma glucose is not accurately predicted by BMI or other obesity measures in the normal and subnormal range, whereas a clearer relationship was observed when overweight and obese persons were included in the study population (Kawano et al., 2005; Kondo, 2006). The magnitude of difference in obesity among our subjects might not be large enough to produce sufficient statistical power. In women, an unexpected inverse relationship between AFI and high glucose was observed. The same inverse relationship was also ascertained by a significant unadjusted correlation coefficient between AFI and the plasma glucose concentration in women $(r=-0.12)$ but not in men $(p=0.5)$. Although a clear underlying mechanism to explain this relationship remains unknown, we speculated there might be a U- or J-shaped relationship between the plasma glucose concentration and AFI, and the result of our study provided only a segment of the overall picture. Albeit Tamakoshi et al. (2003) revealed a U-shaped association between the plasma glucose level and white blood cell count, which is known to indicate a state of low-grade inflammation, the confirmation of our speculation requires a larger sample with a broader spectrum of anthropometric and metabolic characteristics. 
While hyperuricemia is proposed to be part of the metabolic syndrome according to the definition of the World Health Organization (Zimmet et al., 2001), significant relationships between high UA and the obesity index determined by anthropometric or ultrasonographic methods were not confirmed in Model-2. This finding is also unexpected because the recent study by Hikita et al. (2007) indicated that serum uric acid was significantly correlated with both the visceral and subcutaneous fat area quantified by the CT scanning of 508 male industrial workers in Japan. However, they studied younger subjects (mean: 47.5 years, range in age: 24-68) than ours, and failed to model covariates. For now, additional and careful consideration for determining the usefulness of ultrasonographic methods for assessing hyperuricemic risk is required.

This study suggests that the ultrasonographic method would provide an advantage over CT scanning in terms of its costeffectiveness and convenience. Use of skinfold thickness also represents a useful method of subcutaneous fat determination. However, this instrument seems to be subject to errors arising from the possible deformity of bulk tissue during reading or varying elastic properties of both fat and skin (Booth et al., 1966). Cross-sectional data limit this study's results. It is possible that both long-term exposure to a different cultural environment and short-term rapid shift in lifestyle factors impact body composition (Schwingel et al., 2007; Zsolt et al., 2007). Longitudinal observations allow inferences regarding causal relationships between abdominal fat accumulation and the development of obesity-associated metabolic risk factors.

Acknowledgement Financial support for this research was provided by a Grant-in-Aid for Scientific Research (C)(2) (No. 11670368) from the Ministry of Education, Culture, Sports, Science and Technology of Japan.

\section{References}

Armellini F, Zamboni M, Rigo L, Todesco T, BergamoAndreis IA, Procacci C, Bosello O (1990) The contribution of sonography to the measurement of intra-abdominal fat. $\mathrm{J}$ Clin Ultrasound 18: 563-567

Booth RA, Goddard BA, Paton A (1966) Measurement of fat thickness in man: a comparison of ultrasound, Harpenden calipers and electrical conductivity. Br J Nutr 20: 719-725

Blair D, Habicht JP, Sims EA, Sylwester D, Abraham S (1984) Evidence for an increased risk for hypertension with centrally located body fat and the effect of race and sex on this risk. Am J Epidemiol 119: 526-540

Davison AC, Hinkley DV (2003) Bootstrap methods and their application. Cambridge University Press, Cambridge

Expert Panel on Detection Evaluation and Treatment of High Blood Cholesterol in Adults (2001) Executive Summary of the Third Report of the National Cholesterol Education Program (NCEP) Expert Panel on Detection, Evaluation, and Treatment of High Blood Cholesterol in Adults (Adult
Treatment Panel III). JAMA 285: 2486-2497

Ferreira I, Snijder MB, Twisk JW, van Mechelen W, Kemper HC, Seidell JC, Stehouwer CD (2004) Central fat mass versus peripheral fat and lean mass: opposite (adverse versus favorable) associations with arterial stiffness? The Amsterdam Growth and Health Longitudinal Study. J Clin Endocrinol Metab 89: 2632-2639

Fletcher RH, Fletcher SW, Wagner EH (1999) Clinical epidemiology: the essentials. Lippincott Williams \& Wilkins, Baltimore

Folsom AR, Kaye SA, Sellers TA, Hong CP, Cerhan JR, Potter JD, Prineas RJ (1993) Body fat distribution and 5-year risk of death in older women. JAMA 269: 483-487

Fujioka S, Matsuzawa Y, Tokunaga K, Tarui S (1987) Contribution of intra-abdominal fat accumulation to the impairment of glucose and lipid metabolism in human obesity. Metabolism 36: 54-59

Hartz AJ, Rupley DC, Rimm AA (1984) The association of girth measurements with disease in 32,856 women. Am J Epidemiol 119: 71-80

Hikita M, Ohno I, Mori Y, Ichida K, Yokose T, Hosoya T (2007) Relationship between hyperuricemia and body fat distribution. Intern Med 46: 1353-1358

Katzel LI, Busby-Whitehead MJ, Goldberg AP (1993) Adverse effects of abdominal obesity on lipoprotein lipids in healthy older men. Exp Gerontol 28: 411-420

Kawamoto R, Kajiwara T, Oka Y, Takagi Y (2002) Association between abdominal wall fat index and carotid atherosclerosis in women. J Atheroscler Thromb 9: 213-218

Kawano Y, Suzuki K, Mikami S, Kubota Y, Nagasawa N, Sato F (2005) Use of health checkup data for comparison of relative risks in the association between BMI and metabolic complications among middle-aged and elderly populations. Study of Obesity 11: 30-37 [In Japanese]

Kissebah AH (1996) Intra-abdominal fat: is it a major factor in developing diabetes and coronary artery disease? Diabetes Res Clin Pract suppl: 25-30

Kissebah AH, Vydelingum N, Murray R, Evans DJ, Hartz AJ, Kalkhoff RK, Adams PW (1982) Relation of body fat distribution to metabolic complications of obesity. J Clin Endocrinol Metab 54: 254-260

Kondo T (2006) Implication of ultrasonographic use for assessment of abdominal fat accumulation in predicting atherogenic risk. Study of Obesity 12: 40-46 [In Japanese]

Kvist H, Chowdhury B, Grangard U, Tylen U, Sjostrom L (1988) Total and visceral adipose-tissue volumes derived from measurements with computed tomography in adult men and women: predictive equations. Am J Clin Nutr 48: 1351-1361

Leite CC, Wajchenberg BL, Radominski R, Matsuda D, Cerri GG, Halpern A (2002) Intra-abdominal thickness by ultrasonography to predict risk factors for cardiovascular disease and its correlation with anthropometric measurements. Metabolism 51: 1034-1040

Marin P, Andersson B, Ottosson M, Olbe L, Chowdhury B, 
Kvist H, Holm G, Sjostrom L, Bjorntorp P (1992) The morphology and metabolism of intraabdominal adipose tissue in men. Metabolism 41: 1242-1248

Michels KB, Greenland S, Rosner BA (1998) Does body mass index adequately capture the relation of body composition and body size to health outcomes? Am J Epidemiol 147: $167-172$

Park HS, Oh SW, Cho SI, Choi WH, Kim YS (2004) The metabolic syndrome and associated lifestyle factors among South Korean adults. Int J Epidemiol 33: 328-336

Ross R, Rissanen J, Hudson R (1996) Sensitivity associated with the identification of visceral adipose tissue levels using waist circumference in men and women: effects of weight loss. Int J Obes Relat Metab Disord 20: 533-538

Schwingel A, Nakata Y, Ito LS, Chodzko-Zajko WJ, Erb CT, Shigematsu R, Oba-Shinjo SM, Matsuo T, Shinjo SK, Uno M, Marie SK, Tanaka K (2007) Central obesity and healthrelated factors among middle-aged men: a comparison among native Japanese and Japanese-Brazilians residing in Brazil and Japan. J Physiol Anthropol 26: 339-347

Shimokata H, Tobin JD, Muller DC, Elahi D, Coon PJ, Andres R (1989a) Studies in the distribution of body fat: I. Effects of age, sex, and obesity. J Gerontol 44: 66-73

Shimokata H, Andres R, Coon PJ, Elahi D, Muller DC, Tobin JD (1989b) Studies in the distribution of body fat. II. Longitudinal effects of change in weight. Int J Obes Relat Metab Disord 13: 455-464

Shimokata H, Muller DC, Andres R (1989c) Studies in the distribution of body fat. III. Effects of cigarette smoking. JAMA 261: 1169-1173

Sniderman AD, Cianflone K, Arner P, Summers LK, Frayn KN (1998) The adipocyte, fatty acid trapping, and atherogenesis. Arterioscler Thromb Vasc Biol 18: 147-151

Sniderman AD, Lamarche B, Tilley J, Seccombe D, Frohlich J (2002) Hypertriglyceridemic hyperapoB in type 2 diabetes. Diabetes Care 25: 579-582

Sniderman AD, Bhopal R, Prabhakaran D, Sarrafzadegan N, Tchernof A (2007) Why might South Asians be so susceptible to central obesity and its atherogenic consequences? The adipose tissue overflow hypothesis. Int J
Epidemiol 36: 220-225

Suzuki R, Watanabe S, Hirai Y, Akiyama K, Nishide T, Matsushima Y, Murayama H, Ohshima H, Shinomiya M, Shirai K, Saito Y (1993) Abdominal wall fat index, estimated by ultrasonography, for assessment of the ratio of visceral fat to subcutaneous fat in the abdomen. Am J Med 95: 309-314

Tamakoshi K, Yatsuya H, Kondo T, Hori Y, Zhang H, Ishikawa M, Murata C, Otsuka R, Zhu S, Toyoshima H (2003) Ushaped association between white blood cell count and fasting plasma glucose level. Diabetes Care 26: 950

Vague J (1956) The degree of masculine differentiation of obesities: a factor determining predisposition to diabetes, atherosclerosis, gout, and uric calculous disease. Am J Clin Nutr 4: 20-34

Yamamoto M, Egusa G, Yamakido M (1997) Association of intraabdominal fat and carotid atherosclerosis in non-obese middle-aged men with normal glucose tolerance. Int $\mathrm{J}$ Obesity 21: 948-951

Zhu S, Wang Z, Heshka S, Heo M, Faith MS, Heymsfield SB (2002) Waist circumference and obesity-associated risk factors among whites in the third National Health and Nutrition Examination Survey: clinical action thresholds. Am J Clin Nutr 76: 743-749

Zimmet P, Alberti KG, Shaw J (2001) Global and societal implications of the diabetes epidemic. Nature 414: 782-787

Zsolt S, Zsófia M, János M, Andreas P, András P, Ildikó V, Ng N, Kumagai S (2007) Changes over four years in body composition and oxygen uptake of young adult males after university graduation. J Physiol Anthropol 26: 437-441

Received: February 27, 2008

Accepted: November 5, 2008

Correspondence to: Takaaki Kondo, Program in Radiological and Medical Laboratory Sciences, Nagoya University Graduate School of Medicine, 1-1-20 Daikominami, Higashiku, Nagoya 461-8673, Japan

Phone: +81-52-719-1561

Fax: +81-52-719-1561

e-mail: taka@met.nagoya-u.ac.jp 